\section{Influence of histocompatibility genes on disease susceptibility and treatment response in patients with relapsing-remit- ting multiple sclerosis treated with interferon $\beta$-1a}

\author{
Shlomo Flechter, ${ }^{1}$ Tirza Klein, ${ }^{2}$ \\ Lea Pollak ${ }^{3}$
}

1Multiple Sclerosis Clinical Research and

Therapy Service, Sackler Faculty of

Medicine, Tel Aviv University, Tel Aviv,

Israel;

2Tissue Typing Unit, Rabin Medical

Center, Beilinson Campus, Sackler Faculty

of Medicine, Tel Aviv University, Israel;

3Department of Neurology Assaf Harofeh

Medical Center, Sackler Faculty of

Medicine, Tel Aviv University, Tel Aviv, Israel

\section{Abstract}

Multiple sclerosis (MS) is the most common, non-traumatic cause of neurological disability in young adults. The aim of this study was to investigate the influence of HLA class II alleles DRB1* and DQB1* on susceptibility to relapsing-remitting (RR) $\mathrm{MS}$ and response to interferon (IFN) $\beta$-1a treatment. A prospective observational study was conducted. Seventeen patients with clinically definite RRMS, attending a tertiary referral center for multiple sclerosis in Israel and receiving treatment with subcutaneous IFN $\beta$-1a, 22 mcg three times weekly were recruited between December 1998 and February 2000 and observed for 12 months. HLA genotyping was performed and clinical characteristics (relapse rate and disability progression) assessed at baseline and after 12 months. HLA data for a healthy control group were also used for comparison. HLA and the success of treatment with IFN $\beta$-1a in this group of RRMS patients were assessed. The frequency of DRB1*03 was six times higher in patients treated with IFN $\beta$-la than in the healthy control group $(\mathrm{n}=100): 29 \%(5 / 17)$ versus 5\% (5/100), respectively. Additionally, DQB1*03 and $\mathrm{DQB1}{ }^{*} 02$ were present in $82 \%$ $(14 / 17)$ and $41 \%(7 / 17)$ of RRMS patients, but in only $33 \%(33 / 100)$ and $18 \%(18 / 100)$ of control patients, respectively. A better response to IFN $\beta$-1a treatment was seen in patients carrying these alleles than in patients without these alleles. Our results indicated that $\mathrm{DRB} 1 * 03$, $\mathrm{DQB1}{ }^{*} 03$ and $\mathrm{DQB} 1{ }^{*} 02$ alleles may contribute to MS susceptibility and IFN $\beta$-1a responsiveness, and warrant further verification in a larger population.

\section{Introduction}

Multiple sclerosis (MS) is the most common, non-traumatic cause of neurological disability in young adults. It is a chronic, inflammatory, demyelinating, immune-mediated, neurodegenerative disease of the central nervous system (CNS) that occurs in genetically susceptible patients and leads to progressive, chronic, neurological disability. The course of the disease is highly variable, with no reliable prognostic signs. MS can be classified as relapsing-remitting (RR), progressive relapsing, secondary progressive, or chronic (or primary) progressive, based on the clinical manifestation of the disease.1-5

Although the etiology of MS remains unknown, genetic and immunological factors have been implicated in its pathophysiology. The consensus that damage to the nervous system is the result of an immunological/ inflammatory process has been the foundation on which current therapy for MS is based and at which future drug development is aimed.6-8

Current therapy for RRMS is aimed towards prevention of relapses, progression of the disease (as expressed by disability accumulation), and new lesion formation in the brain. Corticosteroids are the mainstay of treatment for acute exacerbations and are thought to mediate their effects through the modulation of both the immune system and systemic endocrine function. ${ }^{9}$ It has been claimed that immunosuppressive agents, such as azathioprine, cyclophosphamide and cyclosporin-A, reduce the number of relapses, but there is no consensus on the efficacy of these drugs in RRMS ${ }^{10}$ Other therapies that have been investigated include copolymer-1 and interferon (IFN), which are considered today to be disease-modifying drugs (DMDs) with beneficial effects on relapse rates, disease progression, new lesion formation and the burden of disease (as demonstrated by magnetic resonance imaging [MRI]).11-14

Several studies have indicated that relapsing forms of MS may be associated with a genetic factor in the HLA class II region. Specifically, patients carrying the HLA-DR-DQ haplotypes HLA-DRB1*1501, -DRB5*0101, $\mathrm{DQA1} * 0102$ or $-\mathrm{DQB1}{ }^{*} 0602$ were found to have an increased risk for MS. Indeed, this risk increased by up to three to four times in Western and European countries. ${ }^{15-29}$ However, the influence of these haplotypes on the course of the disease in patients with relapsing MS who are receiving IFN $\beta$-1a treatment remains unknown.

As well as HLA haplotypes, cytokines also play a crucial role in the pathogenesis of MS. They have specific effects on cell communication and behavior, and on cell-to-cell interaction. 7,30
Correspondence: Shlomo Flechter, Multiple Sclerosis Clinical Research and Therapy Service, Assaf Harofeh Medical Center, Zerifin 70300, Israel.

Tel: + 972.8.9779.878 - Fax: +972.8.9779 879

E-mail: shlomofl@asaf.health.gov.il

Key words: genetic polymorphism, HLA alleles, incidence, interferon $\beta$-1a, relapsing-remitting multiple sclerosis.

Acknowledgments: this work was supported by Merck Serono International S.A., an affiliate of Merck KGaA, Darmstadt, Germany The funding organization played no role in the study apart from providing funds. Dr. Shlomo Flechter, the principal investigator, takes full responsibility for the data, the analyses and interpretation, and the conduct of the research. He had full access to all of the data and has the right to publish any and all data, separate and apart from the attitudes of the sponsor.

Contributions: SF, study conception and design, manuscript revision, manuscript final approval; TK, data acquisition, manuscript revision, manuscript final approval; LP data analysis and interpretation, manuscript drafting, manuscript final approval.

Conflict of interest: the authors report no conflicts of interest.

Received for publication: 24 June 2010. Accepted for publication: 9 May 2011.

This work is licensed under a Creative Commons Attribution NonCommercial 3.0 License (CC BYNC 3.0).

(C) Copyright S. Flechter et al., 2011

Licensee PAGEPress, Italy

Neurology International 2011; 3:e5

doi:10.4081/ni.2011.e5

Pro-inflammatory cytokines, such as interleukin (IL)-2, IL-12, tumour necrosis factor (TNF) $\alpha$ and IFN $\gamma$, are produced by Th1 cells and activate immune cells to enhance the expression of adhesion molecules and chemokines. They also render the blood-brain barrier (BBB) more permeable to soluble and cellular components of the immune system, thereby facilitating the development of inflammatory lesions in the CNS.7 Anti-inflammatory cytokines, such as IL-4, -6, -10, -13 and transforming growth factor (TGF) $\beta$, are produced by Th2 cells and can down regulate the inflammatory response.7,30

Cell adhesion molecules (CAMs) also play a key role in the pathogenesis of the inflammatory response in MS. Activated T cells adhere to the microvasculature using CAMs before passing through the endothelial barrier. Once inside the CNS, T cells are activated and an inflammatory response is initiated by cytokine secretion. Adhesion molecules are up regulated, T cells proliferate and additional effectors 
cells are recruited, leading to the destruction of myelin and oligodendrocytes. Up-regulation of adhesion molecules, in particular vascular cell adhesion molecule-1 and intercellular adhesion molecule-1 (ICAM-1), has been demonstrated in the brain of patients with MS, especially in the proximity of inflammatory lesions. ${ }^{31,32}$ Increased levels of soluble ICAM-1 have also been found in the blood of clinically active patients with relapse or MRI activity, and were found to correlate with increased TNF- $\alpha$, cerebrospinal fluid pleocytosis and BBB damage. .33-35 $^{-35}$

The aims of this study were two-fold. The first objective was to compare the frequency of HLA class II alleles DRB1* and DQB1* in RRMS patients, attending a centre in Israel, with that in healthy individuals. The second objective was to assess the influence of these HLA alleles on responsiveness to IFN $\beta$-1a (number of relapses and disability, as assessed by the Expanded Disability Status Scale [EDSS] score) in patients with RRMS. The effects of treatment on serum expression of various pro-inflammatory and anti-inflammatory cytokines, and adhesion molecules (TNF$\alpha$, IFN- $\gamma$, ICAM-1, IL-4, IL-10 and TGF $\beta$ ) were also assessed in these patients.

\section{Materials and Methods}

\section{Patients}

This was an observational open study designed for one year of follow-up. Consecutive patients with RRMS receiving treatment with subcutaneous IFN $\beta-1 \mathrm{a}, 22 \mathrm{mcg}$ three times weekly at the centre were recruited between December 1998 and February 2000. All patients enrolled in the study had clinically definite MS, according to Poser's criteria, ${ }^{36}$ specifically RRMS. The patients were diseasemodifying-drug-naive patients. All patients gave informed consent prior to their inclusion in the study by signing an informed consent form and the study was approved by the Local Ethics Committee at Assaf Harofeh Medical Center, and therefore the study was performed in accordance with the ethical standards laid down in the 1964 Declaration of Helsinki.

HLA data for a control group were also used for comparison. This control group comprised healthy individuals from the general population with information stored on a central database held by the Tissue Typing Unit at Rabin Medical Center, affiliated to the University of Tel Aviv, Israel.

\section{Assessments}

Clinical characteristics (relapse rate, EDSS score) were assessed at baseline and after 12 months of treatment. Response to treatment from baseline to 12 months was rated as 'high' (no relapses; with improvement of 1 point in EDSS), medium ( $\geq 50 \%$ reduction in relapse rate; improvement of $1 / 2$ point or no change in EDSS) or low ( $<50 \%$ reduction in relapse rate; deterioration in EDSS). HLA genotyping was conducted at the start of the study. Serum biomarker expression (ICAM-I, TNF- $\alpha$, TGF $\beta$, IL10, IFN- $\gamma$ ) was measured at baseline and at 3 monthly intervals throughout the 12 -month study.

\section{HLA genotyping}

HLA genotyping (class II, DRB1*/DQB1* alleles) was performed on blood samples by low-resolution analysis using polymerase chain reaction (PCR) amplification and sequence-specific primers. Tissue typing for HLA was performed using the National Institute of Health microlymphocytotoxicity technique. DNA-based low-medium resolution analysis for $\mathrm{DRB} 1$ * and $\mathrm{DQB} 1 *$ alleles was performed using PCR amplification with sequence-specific primers. For those alleles found to have significantly increased frequency, high-resolution analysis was done by means of PCR sequence-specific oligotyping.

\section{Biomarker expression}

Blood serum was collected at 3-monthly intervals and stored at $-70^{\circ} \mathrm{C}$. Levels of cytokines were measured by using Enzyme Linked Immunosorbent Assay (ELISA) (Commercial kits T-cell Diagnostics).

\section{Treatment}

Patients were treated on an out-patient basis. Subcutaneous IFN $\beta$-1a (Rebif ${ }^{\circledR}$, Merck Serono International S.A., Geneva, Switzerland) was administered three times weekly at a dose of $22 \mathrm{mcg}$ (6 MIU) for 12 months.

Table 1. Patient demographics.

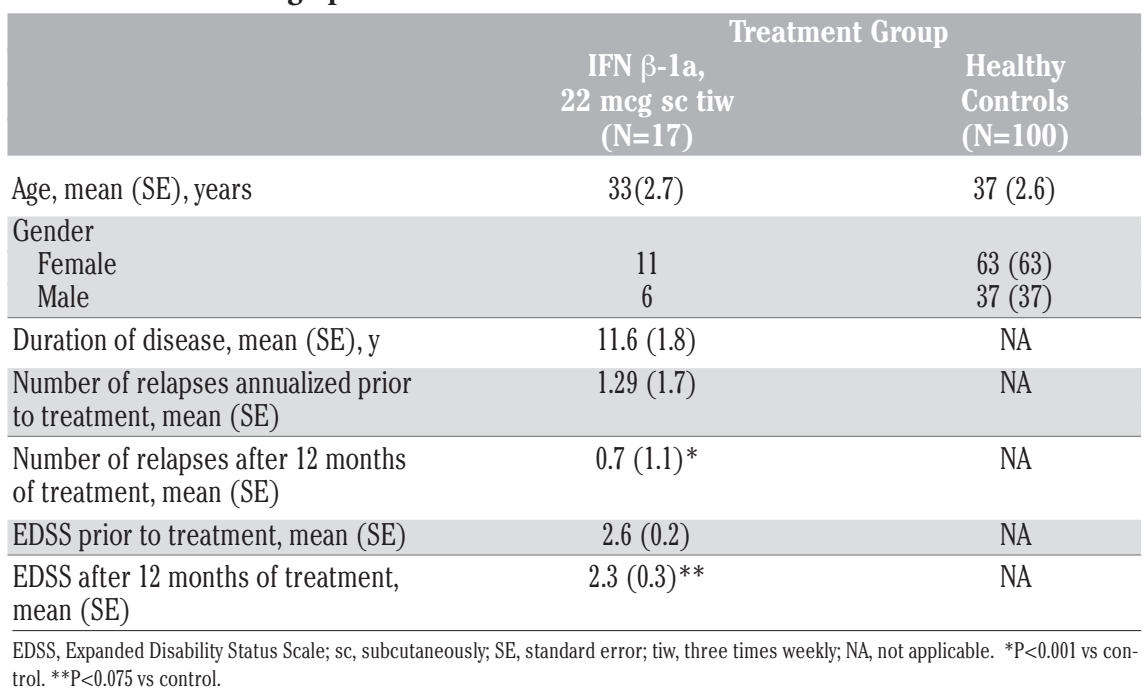

\section{Statistical analysis}

The statistical evaluations were performed by Medistat, a company conducting statistical reviews for medical purposes. The company sponsoring the research did not conduct any statistical evaluations. A paired t-test and nonparametric sign-rank tests were used to test for differences between EDSS scores and the relapse rate before and after 12 months of treatment with IFN $\beta$-1a. A 2-sample t-test and non-parametric test were applied for testing differences between the study groups for quantitative parameters. Spearman's correlation was applied for testing the correlation between the study parameters examined.

All tests applied were 2-tailed, and $\mathrm{P}$ values of $5 \%$ or less were considered statistically significant. Data were analyzed using the SAS software (SAS Institute, Cary, NC, USA).

\section{Results}

\section{Patient characteristics}

A total of 17 patients with definite RRMS were included in the RRMS group and data from 100 healthy subjects was used for the control group. Patient demographics for the RRMS group and the healthy control group are presented in Table 1. The majority of patients in the RRMS group originated from Israel $(\mathrm{n}=10)$, two were from Iran, and one each from Belgium, Georgia, Romania, Russia and Ukraine. Patients were either of Ashkenazi $(n=9)$ or Sephardi $(n=8)$ ethnicity.

\section{Treatment response}

Clinical follow-up after 12 months of IFN $\beta$ la treatment demonstrated a statistically significant decrease in the mean number of relapses compared with the annualised rate 2 years pre-baseline 0.7 (1.1) vs 1.29 (1.7 ); trol. ${ }^{* *} \mathrm{P}<0.075$ vs control. 
$\mathrm{P}<0.001$ (Figure 1a). In IFN $\beta$-1a-treated patients, EDSS scores improved in nine patients, remained stable in five and deteriorated in three; mean (SE) EDSS scores decreased from $2.6(0.2)$ pre-treatment to 2.3 (0.3) post-treatment $(\mathrm{P}<0.075)$ (Figure $1 \mathrm{~b})$.

\section{HLA genotyping}

HLA class II genotyping demonstrated that the frequency of $\mathrm{DRB} 1 * 03, \mathrm{DQB} 1^{*} 03$ and DQB1 ${ }^{*} 02$ alleles was significantly higher in the patients with RRMS than in the control group $(\mathrm{P}<0.05)$ (Figure 2). Patients with these HLA alleles demonstrated a better responsiveness to IFN $\beta$-1a treatment than those without the alleles, according to both the number of relapses, and their levels of disability (Figure 3).

\section{Biomarker expression}

Biomarker expression at baseline and changes after treatment are shown in Figure 4. TGF $\beta$ expression showed the greatest effect, with the majority of patients $(12 / 17,71 \%)$ having levels below normal before treatment and the majority of patients $(13 / 17,76 \%)$ showing an increase in TGF $\beta$ levels after treatment.

\section{Discussion}

Understanding of the etiology and pathogenesis of MS has changed over time. Current progress in molecular genetics has contributed to the hypothesis that MS should be considered as a genetically determined disorder.1,2,6 Indeed, much effort has been made to identify the genetic factors involved in MS. To date, only one factor of confirmed importance has been identified: the HLA class II region on the short arm of chromosome 6.15-29 Studies have reported that carrying the HLA class II DR-DQ haplotypes: DR15, DQ6 and DW2, known in genomic terminology as HLA-DRBI*1501, DRB5*0101, DQA1*0102, DQB1*0602, increases the risk for MS by three to four times in Western and European populations. ${ }^{15-29}$

The prevalence of the HLA-DW2 genotype is high in Europe and North America, but it can be detected in most ethnic groups. Although its frequency is identical in both patients with MS and healthy individuals, it confers a four-fold increase in the risk for MS. A possible application of the HLA association in MS might be that the DW2 haplotype could serve as a prognostic marker; other studies suggest that those individuals with the DW2/DR2 haplotypes might have a worse prognosis than individuals who do not carry these haplotypes. ${ }^{37,38}$

Other HLA haplotypes have also been associated with MS, including DR3 and DQ2, but to a lesser degree than DW2.26,39,40 The DR3 association is with the DR17, DQ2 haplotype, pre-

A

MEAN NO. OF RELAPSES BY PERIOD (N=17)

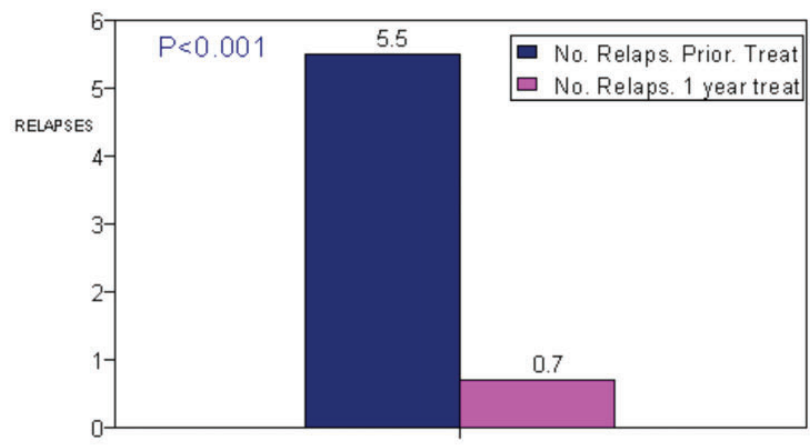

B

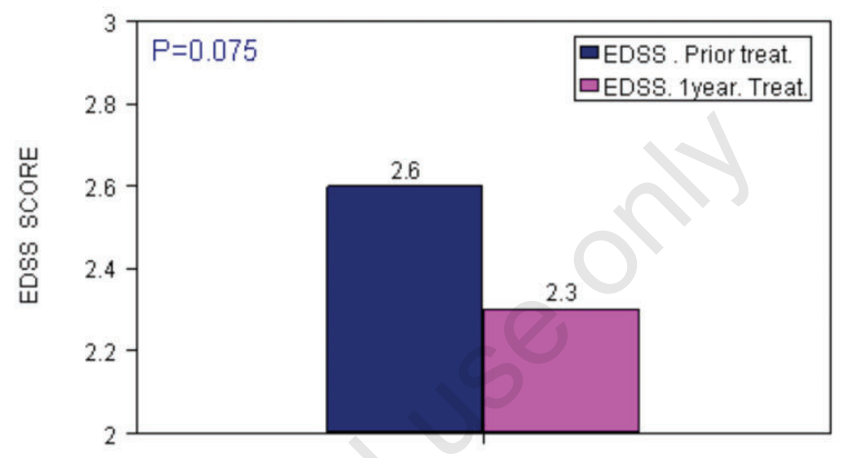

Figure 1. (A) Number of relapses and (B) expanded disability status scale (EDSS) scores before and after 12 months of treatment with interferon beta-1a, $22 \mathrm{mcg}$ (6 MIU) subcutaneously three times weekly.

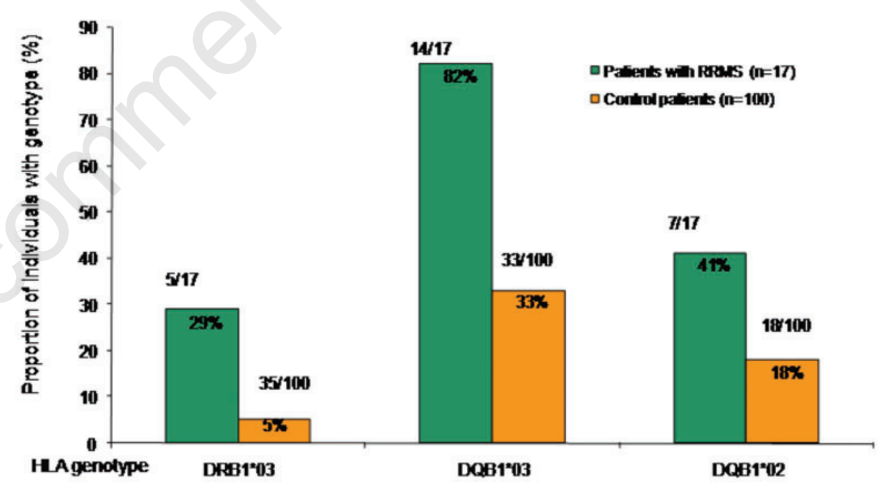

Figure 2. Incidence of human leukocyte antigen (HLA) alleles in patients with relapsingremitting multiple sclerosis (RRMS) and healthy individuals (control group).

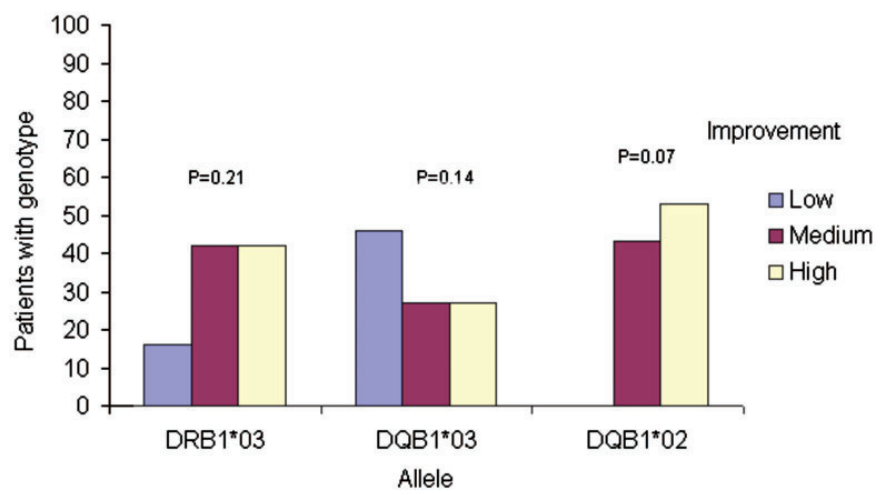

Figure 3. Per centage of patients with each HLA genotype by treatment response, classed by improvement in relapse rate. 

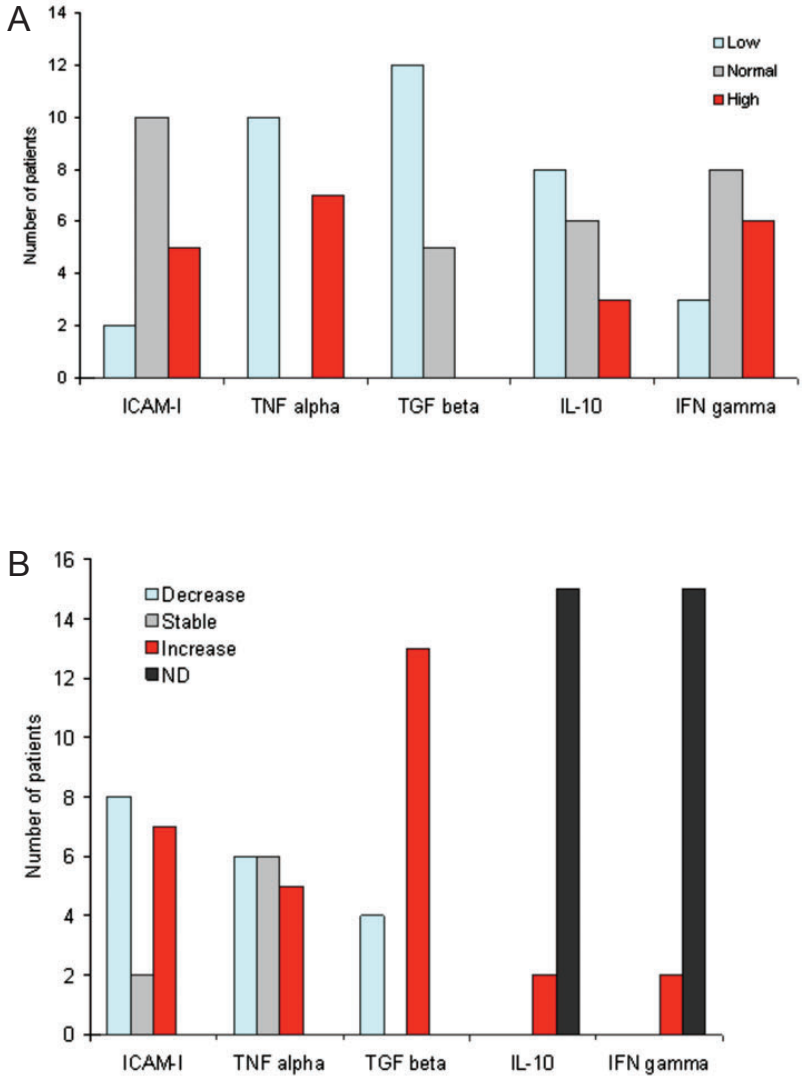

sented as HLA-DRB1*0301, DRB3*0101, DQA1*0501, DQB1*0201.41 In some studies comparing Brazilian MS patients of African origin with ethnically matched controls [17m23], an increased frequency of DQ6 (DQA1*0201, DQB1*0602) in the absence of DR15 was observed. This observation may indicate that the DQ molecule rather than the DR molecule is functionally responsible for the disease-predisposing association in MS. A Sardinian study found DR4 to be increased in patients with MS compared with control individuals. ${ }^{4}$

In our study, we examined the frequency of HLA class II alleles DRB1* and DQB1* and found a higher frequency of $\mathrm{DRB} 1 * 03$, $\mathrm{DQB} 1 * 03$ and $\mathrm{DQB} 1 * 02$ alleles in patients with RRMS compared with the healthy control group. Furthermore, patients with RRMS carrying these alleles showed a better response to IFN $\beta$-1a treatment than those who did not carry these alleles, as was shown. This suggests that the $\mathrm{DRB} 1 * 03, \mathrm{DQB} 1{ }^{*} 03$ and $\mathrm{DQB} 1 * 02$ alleles may be involved in both disease susceptibility and IFN $\beta$-1a response. Although our findings from this small cohort warrant further investigation in a larger population, they indicate that the $\mathrm{DRB} 1 * 03$, $\mathrm{DQB} 1{ }^{*} 03$ and $\mathrm{DQB} 1 * 02$ alleles may have utility as pharmacogenomic markers in RRMS.

As MS is characterized by inflammatory mechanisms, genes encoding cytokines, such as TNF- $\alpha$ and IFN- $\gamma$, are important candidates for genetic markers in MS, particularly as they
Figure 4. Serum biomarker expression levels (A) at baseline and (B) changes after treatment with interferon beta-1a. lation by the end of the study. The complexity and heterogeneity of MS, leads to difficulties in the selection of an appropriate DMD, since the effects of these drugs may differ according to the genetic susceptibility of the patient. The possibility of genetic screening before the initiation of a specific treatment may permit the tailoring of appropriate therapy to the individual patient, so improving the prognosis. The results, which demonstrated a high beneficial effect of the treatment with IFN $\beta$ 1a among the patients carrying these certain alleles, should be judged cautiously, since the number of the patients was small, and more extensive studies, both prospective and retrospective, with a larger number of patients and different disease-modifying drugs, are needed to verify this hypothesis.

\section{References}

1. Bomprezzi R, Ristori G, Cannoni S, et al. MS genetics: New biotechnologies for structural and functional approaches. Int MS J 1999;6:43-9.

2. Sawcer S, Goodfellow PN, Compston A. The genetic analysis of multiple sclerosis. Trends Genet 1997;13:234-9.

3. Wood NW, Sawcer SJ, Kellar-Wood HF, et al. Susceptibility to multiple sclerosis and the immunoglobulin heavy chain variable region. J Neurol 1995;242:677-82.

4. Chataway J, Feakes R, Coraddu F, et al. The genetics of multiple sclerosis: principles, background and updated results of the United Kingdom systematic genome screen. Brain 1998;121:1869-87.

5. Thompson AJ, Kermode AG, Wicks D, et al. Major differences in the dynamics of primary and secondary progressive multiple sclerosis. Ann Neurol 1991;29:53-62.

6. Hillert J, Masterman T. The genetics of multiple sclerosis. In: Cook SD, editor. Handbook of multiple sclerosis, 3rd edn. New York: Marcel Dekker Inc; 2001. p. 3365.

7. Cope AP. Regulation of autoimmunity by pro-inflammatory cytokines. Curr Opin Immunol 1998;10:669-76.

8. Waksman BH. Autoimmunity in demyelinating diseases. Ann N Y Acad Sci 1988;540:13-24.

9. Dijkstra CD, Polman CH, Berkenbosch F. Multiple sclerosis: some possible therapeutic opportunities. Trends Pharmacol Sci 1993;14:124-9.

10. Mitchell G. Update on multiple sclerosis 
therapy. Med Clin North Am 1992;77:23149.

11. Goodin DS, Frohman EM, Garmany GP Jr, et al. Disease modifying therapies in multiple sclerosis: report of the Therapeutics and Technology Assessment Subcommittee of the American Academy of Neurology and the MS Council for Clinical Practice Guidelines. Neurology 2002;58:169-78.

12. Compston A, Coles A. Multiple sclerosis. Lancet 2002;359:1221-31.

13. Harding AE, Sweeney MG, Miller DH, et al. Occurrence of a multiple sclerosis-like illness in women who have a Leber's hereditary optic neuropathy mitochondrial DNA mutation. Brain 1992;115:979-89.

14. Keegan BM, Noseworthy JH. Multiple sclerosis. Ann Rev Med 2002;53:285-302.

15. Yamasaki K, Horiuchi I, Minohara M, et al. HLA-DPB $1 * 0501$-associated opticospinal multiple sclerosis: clinical, neuroimaging and immunogenetic studies. Brain 1999;122:1689-96.

16. Olerup 0, Hillert J. HLA class II-associated genetic susceptibility in multiple sclerosis: a critical evaluation. Tissue Antigens 1991;38:1-15.

17. Marrosu MG, Muntoni F, Murru MR, et al. HLA-DQB1 genotype in Sardinian multiple sclerosis: evidence for a key role of DQB1 ${ }^{*} 0201$ and ${ }^{*} 0302$ alleles. Neurology 1992;42:883-6.

18. Hillert J, Grönning M, Nyland H, et al. An immunogenetic heterogeneity in multiple sclerosis. J Neurol Neurosurg Psychiatry 1992;55:887-90.

19. Haines JL, Ter-Minassian M, Bazyk A, et al. A complete genomic screen for multiple sclerosis underscores a role for the major histocompatability complex. The Multiple Sclerosis Genetics Group. Nat Genet 1996;13:469-71.

20. GAMES; Transatlantic Multiple Sclerosis Genetics Cooperative. A meta-analysis of whole genome linkage screens in multiple sclerosis. J Neuroimmunol 2003;143:3946.

21. Coraddu F, Sawcer S, Feakes R, et al. HLA typing in the United Kingdom multiple sclerosis genome screen. Neurogenetics 1998;2:24-33.

22. Brautbar C, Amar A, Cohen N, et al. HLA-D typing in multiple sclerosis: Israelis tested with European homozygous typing cells. Tissue Antigens 1982;19:189-97.

23. Marcadet A, Massart C, Semana G, et al. Association of class II HLA-DQ beta chain DNA restriction fragments with multiple sclerosis. Immunogenetics 1985;22:93-6.

24. Spurkland A, Ronningen KS, Vandvik B, et al. HLA-DQA1 and HLA-DQB1 genes may jointly determine susceptibility to develop multiple sclerosis. Hum Immunol 1991;30:69-75.

25. Francis DA, Thompson AJ, Brookes P, et al. Multiple sclerosis and HLA: is the susceptibility gene really HLA-DR or -DQ? Hum Immunol 1991;32:119-24.

26. Hillert $\mathrm{J}$, Olerup 0. Multiple sclerosis is associated with genes within or close to the HLA-DR-DQ subregion on a normal DR15,DQ6,Dw2 haplotype. Neurology 1993;43:163-8.

27. Caballero A, Alvés-León S, PapaisAlvarenga R, et al. DQB1*0602 confers genetic susceptibility to multiple sclerosis in Afro-Brazilians. Tissue Antigens 1999;54:524-6.

28. Oturai A, Larsen F, Ryder LP, et al. Linkage and association analysis of susceptibility regions on chromosomes 5 and 6 in 106 Scandinavian sibling pair families with multiple sclerosis. Ann Neurol 1999;46:612-6.

29. Ciusani E, Allen M, Sandberg-Wollheim M, Foli M, et al. Analysis of HLA-class II DQA1, DQB1, DRB1 and DPB1 in Italian multiple sclerosis patients. Eur J Immunogenet 1995;22:171-8.

30. Belardelli F. Role of interferons and other cytokines in the regulation of the immune response. APMIS 1995;103:161-79.

31. Rossler K, Neuchrist C, Kitz K, et al. Expression of leucocyte adhesion molecules at the human blood-brain barrier (BBB). J Neurosci Res 1992;31:365-74.

32. Rieckmann P, Martin S, Weichselbraun I, et al. Serial analysis of circulating adhesion molecules and TNF receptor in serum from patients with multiple sclerosis: cICAM-1 is an indicator for relapse. Neurology 1994;44:2367-72.

33. Hartung HP, Michels M, Reiners K, et al. Soluble ICAM-1 serum levels in multiple sclerosis and viral encephalitis. Neurology 1993;43:2331-5.

34. Sharief MK, Noori MA, Ciardi M, et al. Increased levels of circulating ICAM-1 in serum and cerebrospinal fluid of patients with active multiple sclerosis. Correlation with TNF-alpha and blood-brain barrier damage. J Neuroimmunol 1993;43:15-21.

35. Khoury SJ, Orav EJ, Guttmann CR, et al. Changes in serum levels of ICAM and TNF$\mathrm{R}$ correlate with disease activity in multiple sclerosis. Neurology 1999;53:758-64.

36. Poser CM, Paty DW, Scheinberg L, et al. New diagnostic criteria for multiple sclerosis: guidelines for research protocols. Ann Neurol 1983;13:227-31.

37. Duquette P, Décary F, Pleines J, et al.
Clinical sub-groups of multiple sclerosis in relation to HLA: DR alleles as possible markers of disease progression. Can J Neurol Sci 1985;12:106-10.

38. Engell T, Raun NE, Thomsen M, Platz P. HLA and heterogeneity of multiple sclerosis. Neurology 1982;32:1043-6.

39. Saruhan-Direskeneli G, Esin S, BaykanKurt B, et al. HLA-DR and -DQ associations with multiple sclerosis in Turkey. Hum Immunol 1997;55:59-65.

40. Coraddu F, Reyes-Yanez MP, Parra A, et al. HLA associations with multiple sclerosis in the Canary Islands. J Neuroimmunol 1998;87:130-5.

41. Bodmer JG, Marsh SG, Albert ED, et al. Nomenclature for factors of the HLA system, 1990. Tissue Antigens 1991;37:97104.

42. Marrosu MG, Muntoni F, Murru MR, Spinicci G, Pischedda MP, Goddi F, et al. Sardinian multiple sclerosis is associated with HLA-DR4: a serologic and molecular analysis. Neurology 1988;38:1749-53.

43. He B, Navikas V, Lundahl J, et al. Tumor necrosis factor alpha-308 alleles in multiple sclerosis and optic neuritis. J Neuroimmunol 1995;63:143-7.

44. Weinshenker BG, Wingerchuk DM, Liu Q, et al. Genetic variation in the tumor necrosis factor alpha gene and the outcome of multiple sclerosis. Neurology 1997;49:37885.

45. Selmaj K, Raine CS, Farooq M, et al. Cytokine cytotoxicity against oligodendrocytes. Apoptosis induced by lymphotoxin. J Immunol 1991;147:1522-9.

46. Janeway CA, Travers P. Autoimmunity: Responses to self antigens. In: Janeway CA, Travers P, editors. Immunobiology: the immune system in health and disease, 2nd edn. New York: Garland; 1996. P. 11.

47. Navikas V, Link J, Palasik W, et al. Increased mRNA expression of IL-10 in mononuclear cells in multiple sclerosis and optic neuritis. Scand J Immunol 1995; 41:171-8.

48. Monteyne P, Van Laere V, Marichal R, Sindic CJ. Cytokine mRNA expression in CSF and peripheral blood mononuclear cells in multiple sclerosis: detection by RTPCR without in vitro stimulation. J Neuroimmunol 1997;180:137-42.

49. Comabella M, Balashov $\mathrm{K}$, Issazadeh $\mathrm{S}$, et al. Elevated interleukin-12 in progressive multiple sclerosis correlates with disease activity and is normalized by pulse cyclophosphamide therapy. J Clin Invest 1998;102:671-8. 